\title{
Localized modes in nonlinear binary kagome ribbons
}

\author{
P. P. Beličev, ${ }^{1}$ G. Gligorić, ${ }^{1}$ A. Radosavljević, ${ }^{1}$ A. Maluckov, ${ }^{1}$ M. Stepić, ${ }^{1}$ R. A. Vicencio, ${ }^{2}$ and M. Johansson ${ }^{3}$ \\ ${ }^{1} P^{*}$ Group, Vinča Institute of Nuclear Sciences, University of Belgrade, P.O.B. 522, 11001 Belgrade, Serbia \\ ${ }^{2}$ Departamento de Física, MSI-Nucleus on Advanced Optics, and Center for Optics and Photonics (CEFOP), Facultad de Ciencias, \\ Universidad de Chile, Santiago, Chile \\ ${ }^{3}$ Department of Physics, Chemistry and Biology, Linköping University, SE-581 83 Linköping, Sweden
}

(Received 17 September 2015; published 30 November 2015)

\begin{abstract}
The localized mode propagation in binary nonlinear kagome ribbons is investigated with the premise to ensure controlled light propagation through photonic lattice media. Particularity of the linear system characterized by the dispersionless flat band in the spectrum is the opening of new minigaps due to the "binarism." Together with the presence of nonlinearity, this determines the guiding mode types and properties. Nonlinearity destabilizes the staggered rings found to be nondiffracting in the linear system, but can give rise to dynamically stable ringlike solutions of several types: unstaggered rings, low-power staggered rings, hour-glass-like solutions, and vortex rings with high power. The type of solutions, i.e., the energy and angular momentum circulation through the nonlinear lattice, can be controlled by suitable initial excitation of the ribbon. In addition, by controlling the system "binarism" various localized modes can be generated and guided through the system, owing to the opening of the minigaps in the spectrum. All these findings offer diverse technical possibilities, especially with respect to the high-speed optical communications and high-power lasers.
\end{abstract}

DOI: 10.1103/PhysRevE.92.052916

PACS number(s): 05.45.Yv, 42.65.Tg, 42.82.Et, 63.20.Pw

\section{INTRODUCTION}

Photonic lattices are well-known optical systems for manipulation and control of light propagation. They are constructed of weakly coupled waveguide arrays providing periodic modulation of refractive index in one, two or three dimensions [1]. Characteristics such as periodicity and discrete translational symmetry define appropriate band-gap energy spectra, which dictate the light propagation [2]. Different geometrical configurations of photonic crystals allow the existence of a plethora of various linear and nonlinear phenomena such as Anderson localization [3], controlled generation of interesting interference patterns using photonic Fibonacci structures [4], rogue waves (see [5] and references therein), and PT-symmetry breaking of solitons formed in waveguide necklaces [6], to mention a few. It is known that the introduction of additional periodicity in the uniform lattice pattern opens new gaps within bands of energy spectrum [7]. Newly created stop bands represent energy regions interesting for research because of the possibility of formation of new types of soliton solutions $[8,9]$.

Particular interest nowadays is on flat-band lattices. The mentioned "flat band" is a dispersionless energy band composed of entirely degenerate states, so that any superposition of these states is nondiffracting. Such patterns can be found in condensed matter contexts [10-12], in the context of localization in the presence of magnetic fields or spin-orbit coupling [13], disorder [14,15], and the fractional quantum Hall effect [16,17], as well as in the photonic topological insulators $[18,19]$. Promising possibilities for application of flat-band optical systems emerge from high flat-band degeneracy and high density of states suitable for enhancing light-matter interaction [20]. In general, linear modes of any periodic structure are completely extended. However, flat-band systems allow the formation of very localized eigenmodes which can be viewed as a destructive linear combination of extended linear wave functions.

Recently studied examples of flat-band systems include quasi-one-dimensional (quasi-1D) diamond ladder [14], two- dimensional (2D) Lieb [21-23], and 2D kagome lattices [24-27]. Particularity of the latter systems are, besides linear localized ring modes, one-peak solutions which bifurcate from flat band at zero power threshold in the presence of nonlinearity [27] and propagate without diffraction through the system. Moreover, for the nonlinear kagome 2D lattices, detailed studies in the continuum and discrete limits showed the existence of dynamically stable configurations such as the in-phase hexapole, single-charge six-site vortex on the honeycomb cell, as well as the four-site in-phase-out-of-phase quadrupole and the second-gap in-phase quadrupole on the hourglass cell [26]. In general, the unstable localized solutions of all mentioned types in the discrete case decompose into breathing configurations with fewer populated sites and some phase correlations.

In [27] significant conclusions are derived with respect to moving localized modes, which can be created by a small transverse kick (phase gradient) of an unstable one-peak mode. It is shown that for small kicks, a transiently moving mode appears which is trapped close to the initial position as a symmetry-broken ground state. For stronger kicks, the moving mode propagates over longer distances before being trapped by the lattice.

Our study is based on the kagome geometry [28], which is easily realizable owing to fast-developing fabrication techniques based on nondiffracting beams [24] and photon lithography [29]. This geometry can also be realized in the context of optical lattices for trapping of ultracold atoms $[30,31]$ or metallic ones for obtaining a flat plasmonic band [32]. We here focus our attention on the discrete nonlinear kagome quasi-1D ribbons, which are dimensionally reduced 2D kagome lattices. Motivated by the paper [33], we start with uniform kagome ribbons and investigate the influence of the "binarism" on the formation and dynamical properties of localized eigenmodes bifurcating from the flat band and second band. These "binary" structures are different from previously studied ones, like binary chains composed of 


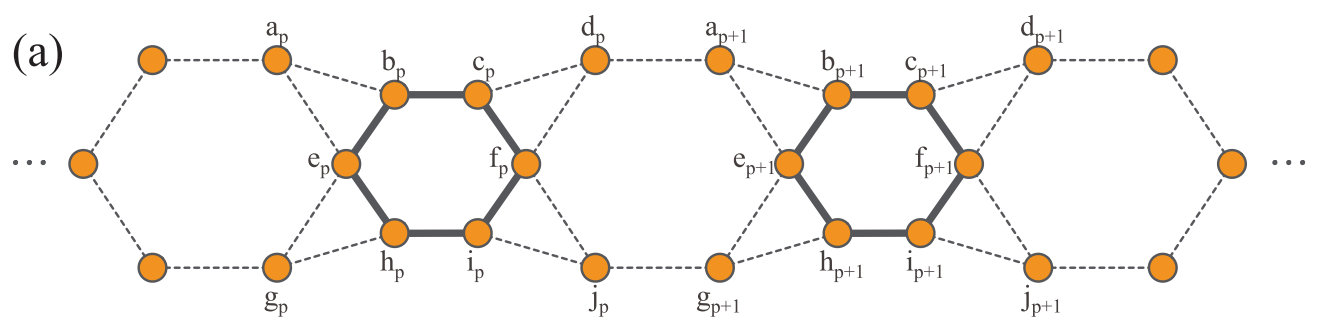

(b)

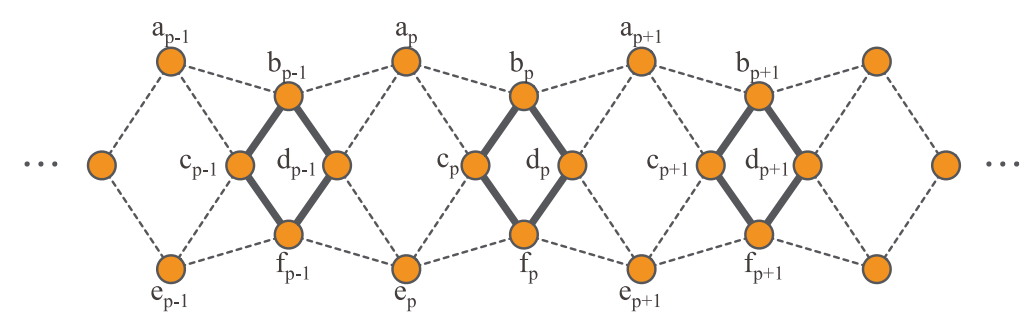

FIG. 1. (Color online) Structure of (a) binary kagome strip and (b) binary kagome ladder. Each characteristic cell comprises (a) 10 sites; (b) six sites, mutually coupled either by $V_{1}$ (dashed line) or by $V_{2}$ (thick line) coupling strength.

dimers as discussed in [34]. Particularity of our systems is the coexistence of discreteness, nonlinearity, and "binarism." Our analysis covers two types of kagome ribbons, i.e., the kagome strip ([33-35]) and the kagome ladder (or three-spin ladder as it is called in [36]), which differ in geometrical pattern. Such structures can be fabricated by dint of the direct femtosecond laser inscription [37-39]. In the case of uniform ribbons, the first type is characterized by the unit cell containing five sites [40], while the other one (kagome ladder) comprises cells made of three sites. We prove that both geometrical patterns support flat band in spectra of 1D binary ribbons and analyze the influence of "binarism" on the properties of these spectra and consequently, on the light localization and its behavior.

The paper starts with defining the mathematical model of the binary nonlinear kagome ribbons based on the discrete Schrödinger equations with cubic nonlinearity in Sec. II. The structure of the corresponding linear spectra is considered in detail. In Sec. III we present and discuss numerical results obtained for two forms of the nonlinear discrete binary kagome ribbons. In addition to the ring structures of the staggered type, which are found to lose stability in the strongly nonlinear binary kagome ribbons, here we found conditions under which it is possible to obtain different types of dynamically stable vortex-ring solutions, the unstaggered ringlike solutions, hourglass solutions inside the semi-infinite gap, and minigap solitons inside the gaps opened due to lattice binarism. Vortex ring solutions, identified in different settings, studied here in the context of orbital momentum carriers, are also suitable for spatial-division multiplexing, actively studied in high-speed optical communications for increasing bandwidth [41-46]. Finally, Sec. IV concludes the paper. The main finding is that by proper initial ribbon excitation and selection of geometric parameters, the light propagation can be controlled. Throughout the paper, we compare our results for binary kagome ribbons with the results for uniform ribbons [33] and 2D kagome lattices [25-27] in order to stress what binarism adds to these systems.

\section{THE MODEL}

The possibility of linear kagome lattices to guide localized modes is indicated by the existence of the dispersionless flat band in the corresponding eigenvalue spectrum. Control of light propagation is here investigated by introducing the nonlinearity and additional periodicity into the $1 \mathrm{D}$ variant of the kagome lattice, i.e., binarism. Two kagome ribbon configurations are considered. The schematic representation of the binary kagome strip with its characteristic cell is shown in Fig. 1(a). Thick solid lines denote $V_{2}$ linear coupling strength between neighboring sites, while dashed ones stand for coupling strength $V_{1}$. This system consists of $n=N / 10$ cells, each cell containing 10 sites. Here, $N$ is the total number of sites (waveguides), and the cells are indexed by $p, 1 \leqslant p \leqslant n$. The second setup, kagome ladder, is depicted in Fig. 1(b). Due to its different geometry, the system consists of $n=N / 6$ cells, each cell containing six sites.

Proposing dimensional reduction (from 2D to $1 \mathrm{D}$ ) in the frame of the coupled-mode theory (CMT), the evolution of the field amplitude in a nonlinear binary kagome strip and ladder may be described by the following set of differential-difference nonlinear Schrödinger equations (DNSE):

$$
i \frac{d \mathbf{u}}{d z}=\tilde{V} \mathbf{u}+\gamma\left(\operatorname{diag}\left(\mathbf{u}^{\dagger}\right) \operatorname{diag}(\mathbf{u})\right) \mathbf{u},
$$

where $\mathbf{u}(z)$ are the $10 n$ or $6 n$ component column vectors with amplitudes of the single guiding modes centered on the individual waveguides in the kagome strip or ladder:

$$
\begin{aligned}
& \mathbf{u}(z)=\left[a_{1} b_{1} c_{1} d_{1} e_{1} f_{1} g_{1} h_{1} i_{1} j_{1} \ldots . . a_{n}\right. \\
& \left.\times b_{n} c_{n} d_{n} e_{n} f_{n} g_{n} h_{n} i_{n} j_{n}\right]^{T}(z), \\
& \mathbf{u}(z)=\left[\begin{array}{llll}
a_{1} & b_{1} & c_{1} & d_{1} e_{1} f_{1} \ldots . . a_{n} b_{n} c_{n} d_{n} e_{n} f_{n}
\end{array}\right]^{T}(z),
\end{aligned}
$$

respectively. The propagation direction is $z$ and the quadratic matrix $\tilde{V}(10 n \times 10 n$-dimensional for the kagome strip, or $6 n \times 6 n$-dimensional for the kagome ladder) includes coupling coefficients which define the linear nearest neighbor 
interactions between components of $\mathbf{u}$ (see Fig. 1 and the appendix), while $\gamma=-1(\gamma=1)$ represents an effective nonlinear defocusing (focusing) cubic parameter. The systems possess two conserved quantities: total power of the localized solution (norm),

$$
P=\mathbf{u}^{\dagger} \mathbf{u}
$$

and the Hamiltonian,

$$
H=\mathbf{u}^{\dagger} \tilde{V} \mathbf{u}+(\gamma / 2) \operatorname{trace}\left(\left(\operatorname{diag}\left(\mathbf{u}^{\dagger}\right) \operatorname{diag}(\mathbf{u})\right)^{2}\right)
$$

The dispersion relations for the kagome strip and ladder can be obtained from the linearized version (i.e., $\gamma=0$ ) of the system of Eq. (1), presuming solutions in the form: $\left[a_{p} b_{p} c_{p} d_{p} e_{p} f_{p} g_{p} h_{p} i_{p} j_{p}\right]=$ [A B C D E F G H I J] $e^{-i \beta z} e^{i k p}$ (kagome strip), and $\left[a_{p} b_{p} c_{p} d_{p} e_{p} f_{p}\right]=\left[\begin{array}{llllll}A & B & C & D & E & F\end{array}\right] e^{-i \beta z} e^{i k p}$ (kagome ladder), where $k$ stands for the transverse Bloch wave vector of the binary lattice and $\beta$ is the propagation constant of the observed mode.

In the case of a uniform kagome strip $\left(V_{1}=V_{2}=V\right)$ the characteristic cell can be reduced to five sites $(a=c, b=$ $d, e=f, g=i, h=j$ ), giving the following set of dispersion relations $[33,40]$ :

$$
\begin{aligned}
& \beta_{1}=V-V \sqrt{3+2 \cos (k / 2),} \\
& \beta_{2}=V+V \sqrt{3+2 \cos (k / 2)}, \\
& \beta_{3}=-2 V \\
& \beta_{4}=V \sqrt{2(1+\cos (k / 2))} \\
& \beta_{5}=-V \sqrt{2(1+\cos (k / 2))} .
\end{aligned}
$$

On the other hand, the dispersion relation of the uniform kagome ladder, which is characterized by a cell with three sites $(a=b, c=d, e=f)$, contains three branches [40]:

$$
\begin{aligned}
& \beta_{1}=2 V(1+\cos (k / 2)), \\
& \beta_{2}=2 V \cos (k / 2), \\
& \beta_{3}=-2 V .
\end{aligned}
$$

Dispersion relations of the uniform kagome strip and ladder are depicted in Figs. 2(a) and 2(b). Both are characterized by the flat band at $\beta=-2(V=1$ in this figure), which is the lowest band in the corresponding spectra. In the case of the kagome strip, the flat band touches the second band at its extremum at the center of the Brillouin zone, causing the degeneration of these states. On the other hand, degeneration of the second band in the kagome ladder happens due to confluence of the flat and second band at the edges of the Brillouin zone. The more complex spectrum in the case of the kagome strip than in the kagome ladder is due to the larger number of sites in the corresponding fundamental block. Thus, the energy spectra of the linear uniform $\left(V_{1}=V_{2}\right)$ $n$-cell kagome strip and ladder is formed of $5 n$ or $3 n$ energy states [40]. There are no gaps inside the spectrum of the uniform kagome ribbons, except semi-infinite ones (vertical lines in Fig. 3).

By decreasing the ratio $V_{1} / V_{2}$, i.e., introducing the binarism, the gaps in the kagome ribbon spectra start to open and grow. As a consequence, the spectrum divides into
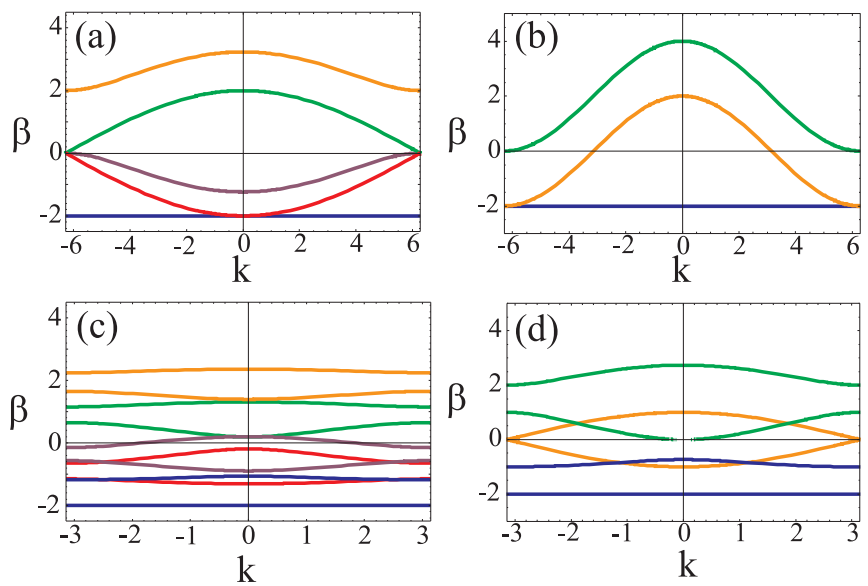

FIG. 2. (Color online) Band structures of 1D (a) uniform kagome strip, (b) uniform kagome ladder, (c) binary kagome strip, and (d) binary kagome ladder. In the case of binary configurations, the values of coupling parameters are set to $V_{1}=0.5$ and $V_{2}=1$.

additional bands, Fig. 3, where newly created minigaps can be distinguished for both systems. The number of minigaps depends on the ratio $V_{1} / V_{2}$ and it is larger for the case of the kagome strip. With decrease of $V_{1}$, bands start to ramble and minigaps become wider. The eigenvalue analysis
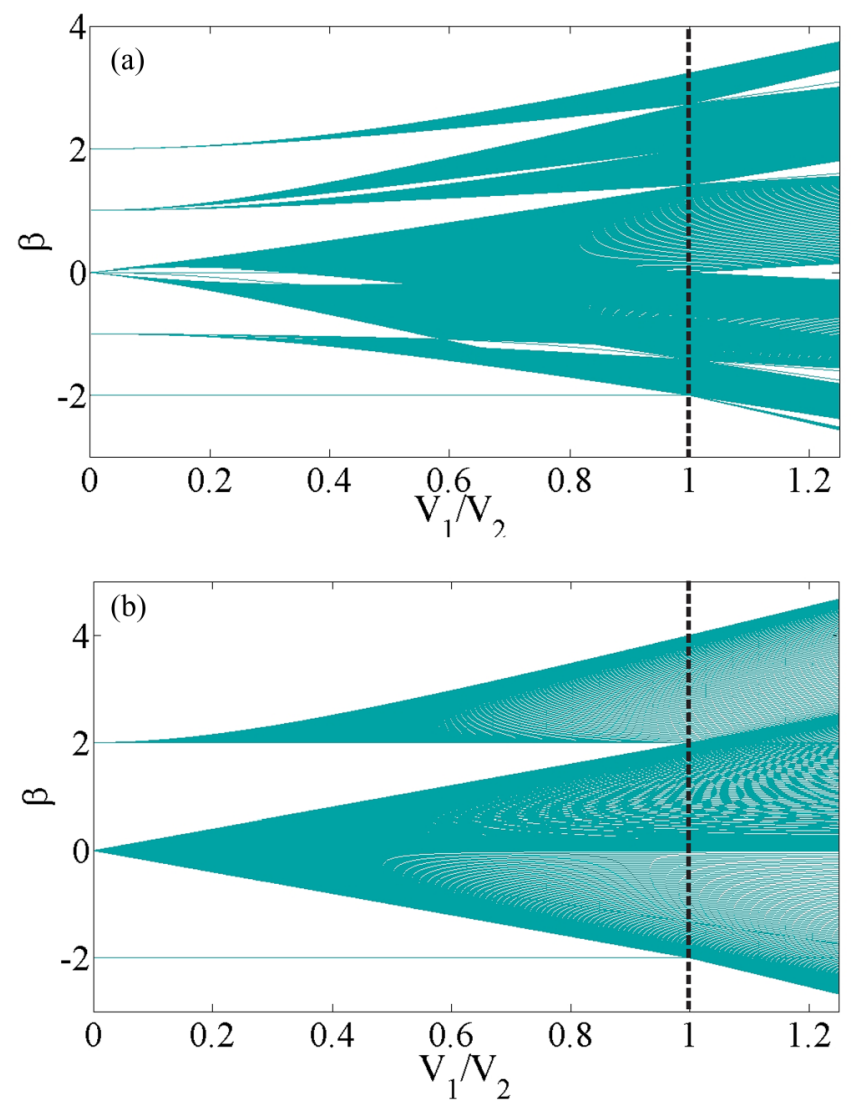

FIG. 3. (Color online) Influence of $V_{1}$ coupling parameter on band structures of 1D binary kagome strip (a) and binary kagome ladder (b). Second coupling parameter $V_{2}$ is fixed to one. Dashed vertical lines mark the case of uniform lattice for which only semi-infinite gaps exist. 
showed that a minigap opens between the flat and the second band for every ratio $V_{1} / V_{2}<1$ in both configurations. In the limit of "independent" cells $\left(V_{1}=0\right)$ the spectrum is totally degenerated and consists of five (three) separate lines. On the other hand, with increase of $V_{1} / V_{2}>1$ the flat band mixes with the second band.

A significant finding is that the flat band exists for the whole range of $V_{1} / V_{2}$. Due to the additional periodicity, the original flat band found in the uniform ribbon splits into two bands, represented by blue (darkest gray) lines in Figs. 2(c) and 2(d). The lower branch remains flat, while the upper one is dispersive. The first minigap opens above the flat band. Moreover, the higher bands split as well, causing the formation of additional minigaps. The shape of these bands and the size of minigaps are functions of the ratio $V_{1} / V_{2}$ [Figs. 3(a) and 3(b)].

Both linear uniform kagome ribbons can guide closed six(four-) node staggered ringlike localized modes, as well as any linear combination of them. These are eigenmodes of the system which can be easily shown analytically by simple substitution of the corresponding amplitudes of modes in the linear version of the stationary form of model Eq. (1). They are characterized by alternation of the sign of the real part of the amplitude in successive sites. The hourglass localized solutions, which can be found to exist in nonlinear kagome ribbons inside the semi-infinite gap, in the linear limit can be represented as a linear combination of two neighboring staggered rings.

Due to the splitting of the flat band, half of the original number of flat-band states found in the uniform ribbon stay in the flat band of the binary lattice at $\beta=-2$ [lower blue lines in Figs. 2(c) and 2(d)]. These modes are here referred to as "strong" rings, since they are localized on strongly coupled sites coupled by $V_{2}$. The other half of solutions corresponds to the upper blue (darkest gray) band which is branched out from the flat band and these modes are formed on "weak" rings of the ribbon, i.e., on rings coupled by $V_{1}$.

Additionally, the flat band of the binary lattice is more isolated from the other bands in the spectrum so the ring localized solutions are more robust on the imperfections in the system than in the case of uniform ribbon, which has been confirmed through our simulations. Linear modes belonging to other bands are found to be completely extended $[47,48]$.

\section{NONLINEAR LOCALIZED MODES IN KAGOME RIBBONS}

The main purpose of our study is to consider the possibility of creating dynamically stable nonlinear localized modes in binary kagome ribbons. Such modes can be viewed as active energy (and momentum) carriers through the system, which are interesting in the context of optoelectronics, photonic communications, etc. The question of how solitons in the uniform 2D kagome lattice with defocusing nonlinearity may bifurcate from flat-band linear modes to the lower semi-infinite gap is addressed in [27]. On the other hand, the behavior of solitons generated in the upper semi-infinite gap in uniform kagome ribbons with focusing nonlinearity was discussed in [33]. Our interests are related to the dynamics of nonlinear solutions which may be found around the flat band. Therefore, we consider in detail dynamically stable localized modes in ribbons with a defocusing type of nonlinearity $(\gamma=-1)$ in semi-inifinite and the first minigap, as well as those generated in ribbons with focusing nonlinearity $(\gamma=1)$ in the first minigap.

The numerical procedure used in our research to find stationary solutions of model Eq. (1) is based on the modified Powell minimization method [49]. As initial guesses to find numerically exact nonlinear solutions, we set appropriate types (shapes) of the specific sought-after solutions. Hourglass and staggered-ring solutions may be obtained from exact solutions in the linear limit, and other solutions from exact solutions in the limit of zero coupling. The convergence tolerance in the performed numerics was set as $10^{-8}$. The stability of the obtained localized solutions is checked by performing the linear stability analysis (LSA) [49] and direct numerical simulation of the mode propagation in the presence of small fluctuations. The last are numerically modelled via the sixth-order Runge-Kutta numerical procedure [50] by adding small random amplitude perturbations to the initial stationary solutions. The conservation of the total power is checked in each calculation step. Additional checking of the compact mode propagation is performed by calculating the effective localization volume measured via the participation number $R$ [15]:

$$
R=\frac{P^{2}}{\mathbf{u}^{\dagger} \mathbf{u} \mathbf{u}^{\dagger} \mathbf{u}} .
$$

The results presented in this paper are collected presuming $V_{2}=1$, while the value of $V_{1}$ was varied in the range from 0 to 1 . The nonlinearity is directly related with the power $P$ of each solution. The total number of lattice cells used in most of the simulations is $n=20$.

\section{A. Localized solutions in semi-infinite gap}

For both kagome configurations (kagome strip and ladder with defocusing nonlinearity), we found three types of nonlinear ringlike modes to exist inside the semi-infinite gap depending on the value of topological charge $S$, and an hourglass solution [33]. The parameter $S$ is defined as the total change of the phase along a closed curve surrounding the center of the ring, divided by $2 \pi$. The ringlike solutions, i.e., solutions with significant amplitude at six (kagome strip) or four sites (kagome ladder) inside one cell can be classified as unstaggered rings (adjacent sites in-phase) with $S=0$, staggered rings with $S=M / 2$ (which exist in the linear case, too), and vortex rings with $S \neq 0, M / 2(M=6$ and $M=4$, for kagome strip and ladder, respectively). Moreover, in the binary case $\left(V_{1} / V_{2} \neq 1\right)$ each nonlinear ringlike solution can be realized in a "weak" or "strong" variant depending on the intersite coupling strength formed between sites coupled by $V_{1}$ ("weak") or between sites coupled by $V_{2}$ ("strong").

The calculations have shown that the "weak" staggered-ring branch appears in the first minigap and can be found to exist in the lower semi-infinite gap, too, in the case of defocusing nonlinearity. However, all these solutions are unstable and thus will not be considered in the following. So, the prefix "strong" in "strong" staggered rings will be omitted.

The staggered nonlinear ring solution's branch originates from the flat band. The LSA and direct numerical simulations 

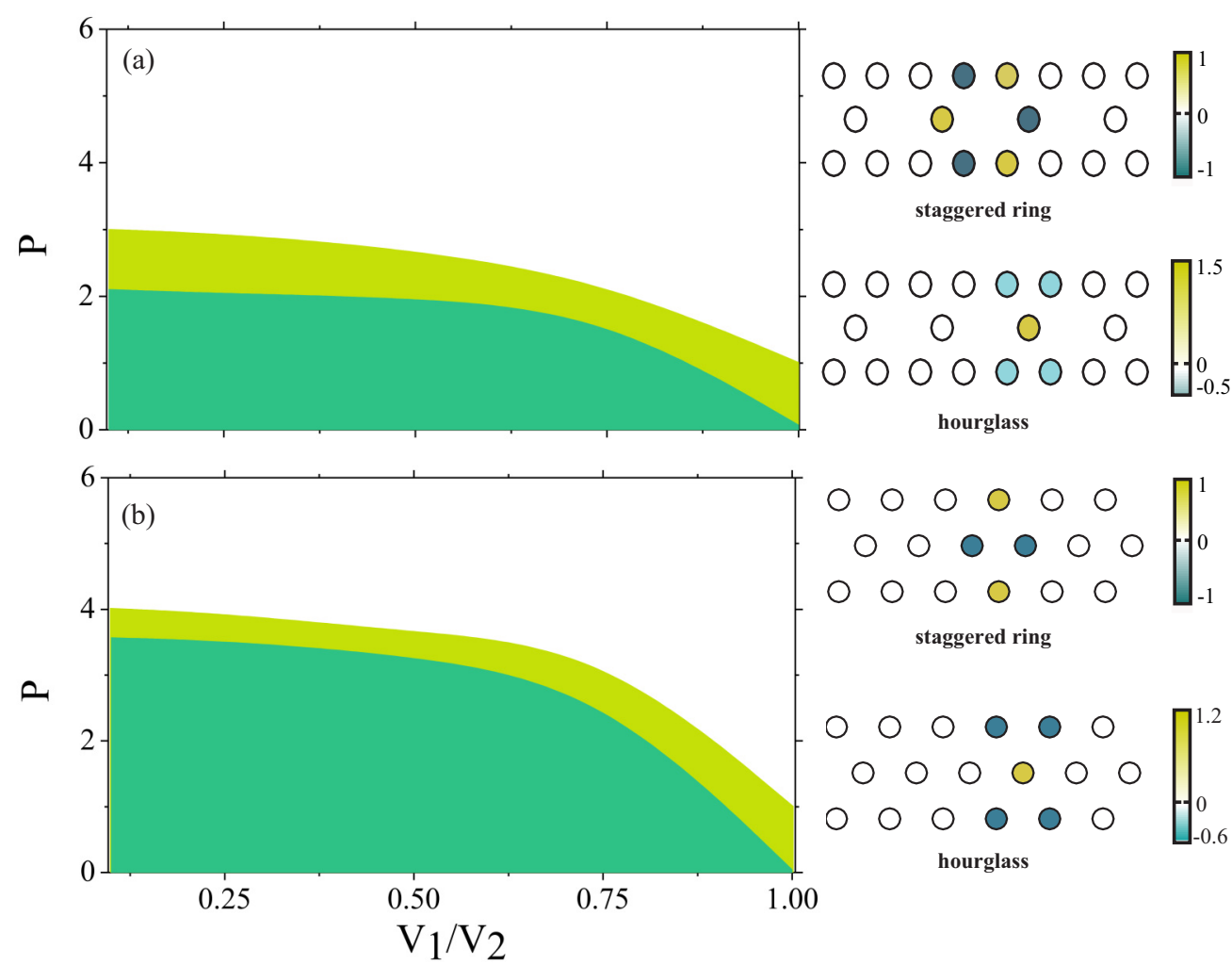

FIG. 4. (Color online) Stability diagrams ( $P$ vs $\left.V_{1} / V_{2}\right)$ of (strong) staggered rings and hourglass solutions in (a) kagome strip and (b) kagome ladder. In the greenish (dark gray) region only stable staggered rings exist, in the yellowish (light gray) region are the stable ring and unstable hourglass solutions, while in the white region the unstable ring and stable hourglass solutions are found. Amplitudes' profiles of certain modes (kagome strip, strong staggered ring $\beta=-3, P=6$; hourglass, $\beta=-3, P=2.61$; kagome ladder, strong staggered ring $\beta=-3, P=4$; hourglass, $\beta=-3, P=2.56$ ) are shown on right panels and correspond to the case of uniform ribbons.

imply a narrow range of $\beta$ (or $P$ ) in the semi-infinite gap for which stable propagation of solutions from this branch occurs. It should be noted that the binarism increases the stability range of staggered modes (see Fig. 4). Examples of the real part of amplitude profiles of staggered ring solutions are illustrated in corresponding panels right from the stability diagrams. The stability region for the kagome strip and ladder is represented by greenish (dark gray) and yellowish (light gray) colors. The observed properties of the staggered rings are in accordance with results published in [26,27] for the 2D kagome lattice where nonlinear modes bifurcate from the flat band without excitation threshold $(P=0)$. Actually, the ring type of fundamental modes is "a nonlinear continuation" of the flat-band six- or four-peak ring modes with exactly the same properties, only with a frequency shift related to the power as $P=6(\beta+2) / \gamma$ (strip) and $P=4(\beta+2) / \gamma$ (ladder), respectively. Therefore, we can conclude that in the presence of defocusing nonlinearity the existence region of staggered rings extends to the semi-infinite gap, while the dynamical stability is observed only for rings in the narrow area close to the flat band. These modes are characterized by small normalized power (however, as gamma is generally very small, real power can be actually quite high). In the white colored part of the existence region in Fig. 4, such solutions are exponentially unstable.

Concerning the hourglass solutions in uniform ribbons, in the linear limit these modes consist of two neighboring rings with one common site (single-peak solution in [27]). In the presence of defocusing nonlinearity their existence region is moved to the semi-infinite gap. Moreover, the nonlinearity offers the possibility of creating hourglass modes in binary ribbons by properly adjusting the shape of the initial light beam excitation in the parameter regions corresponding to the semi-infinite gaps, as shown in Figs. 4(a) and 4(b). Except for the case of uniform lattice, there is always a certain power threshold necessary for their formation [upper bound of the greenish (dark gray) region in Fig. 4]. The yellowish (light gray) region in the stability diagram depicts the parameter area in which hourglass modes are unstable. In white regions hourglass solutions are stable. Furthermore, binarism is responsible for asymmetry of the newly formed hourglass modes.

In addition, the stability exchange between the staggered ring and hourglass modes is observed in a region of small modes' power as depicted in Fig. 4 [border line between the yellowish (light gray) and white region]. Similarly to the 2D kagome lattice [27], in the kagome strip there is generally a very narrow region where both modes are unstable and thus the borders between the yellowish (light gray) and white areas are not perfectly sharp. In this area the calculations have shown that the destabilization of the staggered ring branch is accompanied by birth of the stable two-peak solution branch. This mode reminds one of the intermediate solution in the 2D kagome case [27]. The existence curve and the profile 

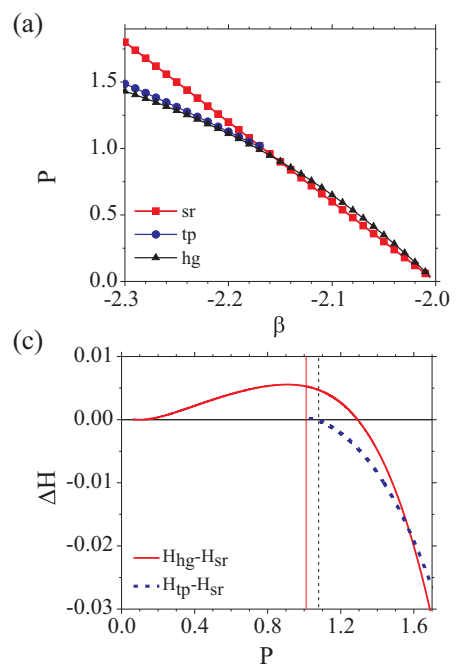

(b)
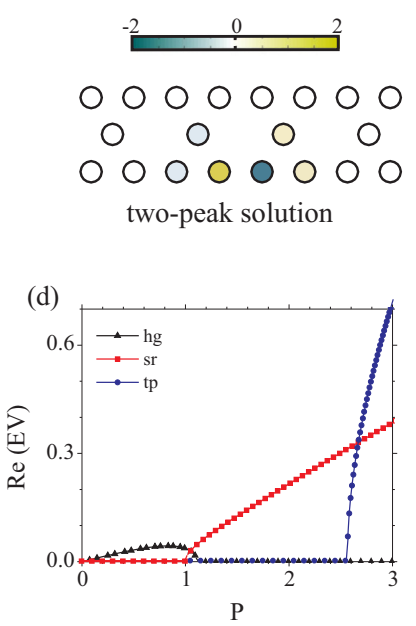

FIG. 5. (Color online) Uniform kagome strip configuration. (a) $P$ vs $\beta$ curves for staggered ring (red line with squares), two-peak solution (blue line with circles), and hourglass (black line with triangles) solutions; (b) amplitude profile of tp solution; (c) $\Delta H$ vs $P$ curve (solid red for $H_{h g}-H_{s r}$ and dashed blue line for $H_{t p}-H_{s r}$ ), solid red vertical line represents the border from which the staggered rings become unstable, and black dashed line the border from which the hourglass solutions become stable; (d) the largest real eigenvalues vs $P$ for staggered ring (red line with squares), two-peak solution (blue line with circles), and hourglass (black line with triangles) solutions.

of the two-peak solution is illustrated in Figs. 5(a) and 5(b), respectively. This mode is bonded at the "edge" of the ring formed of sites coupled via $V_{2}$ (Fig. 1). Besides the two-peak solution branch, we plotted existing curves of staggered ring and hourglass solutions found in the uniform kagome strip [Fig. 5(a)]. In the region of small powers hourglass, two-peak, and staggered ring solutions carry approximately the same power for the fixed propagation constant $\beta$. For modes in this area we calculated the difference of the corresponding Hamiltonians $\Delta H(P)=H_{h g}(P)-H_{s r}(P)$ and $\Delta H(P)=H_{t p}(P)-H_{s r}(P)$, which is illustrated in Fig. 5(c). Here, hg, sr, and tp stand for hourglass, staggered ring, and two-peak solutions, respectively. The staggered ring solution corresponds to the ground state bifurcating from the linear band when $P=0$. The area bounded with vertical solid red and dashed black lines corresponds to the region where both staggered ring and hourglass solutions are characterized by the exponential instability in the LSA, as shown in Fig. 5(d). When staggered ring solutions lose their stability $(P=1.008)$, the existence of stable two-peak solutions is found. At point $P=$ 1.14 the bistability region, where both two-peak and hourglass solutions are stable, opens. The value of the input power determines the branch "chosen" by the system in the bistability region. For example, in the uniform lattice the smaller energy is associated with the hourglass after $P \approx 1.55$. The two-peak branch exists for high powers, too, losing stability at $P \approx 2.4$. Notice that the two-peak solution branch, which is born from the staggered ring branch and is characterized by the narrow stability window, has been found in all binary kagome strip lattices, i.e., for all ratios $V_{1} / V_{2}$.
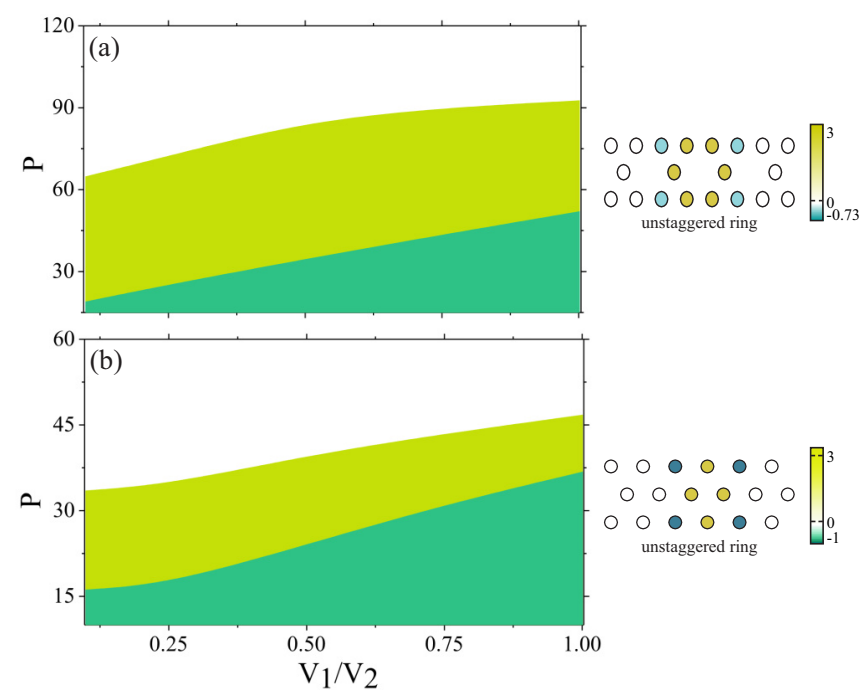

FIG. 6. (Color online) Stability diagrams ( $P$ vs $V_{1} / V_{2}$ ) of strong unstaggered ring solutions in (a) kagome strip and (b) kagome ladder. Greenish (dark gray), yellowish (light gray), and white regions correspond to the region where unstaggered rings do not exist, appear as unstable solutions, and are stable propagating modes, respectively. Amplitudes' profiles of certain modes (kagome strip, $\beta=-10, P=$ 72.45; kagome ladder, $\beta=-10, P=53.2$ ) are shown on right panels and correspond to the case of uniform ribbons.

Besides staggered rings, two variants of the unstaggered ring solutions, "weak" and "strong," are found to exist in the semi-infinite gap, too. The existence branch of "strong" unstaggered rings, with power monotonously increasing with $|\beta|$, appears inside the semi-infinite gap with a certain threshold power value, which is indirectly represented via the stability diagrams in Fig. 6 [in greenish (dark gray) area "strong" unstaggered rings do not exist]. The yellowish (light gray) region represents a parameter area near the appearance boundary in which "strong" unstaggered rings are oscillatorily unstable. As in the 2D kagome systems [26], stable unstaggered ring solutions were also found to exist in the kagome strip and ladder (white area in Fig. 6). Amplitude profiles of unstaggered ring modes are shown within corresponding panels on the right side of the figure. Results showed that the stability region of these solutions increases with the decrease of ratio $V_{1} / V_{2}$. Therefore, the introduced binarism increases the stability regime of unstaggered ring modes. Qualitatively the same trend has been observed for the "weak" variant, too, except that the power thresholds necessary for formation and their stable propagation are lower than in the case of "strong" modes.

The third type of stable localized ring modes found to exist in the semi-infinite gap are vortex solutions. Again, there is a threshold power for bounding vortex in the kagome cell independently where they are created, i.e., between sites coupled with $V_{1}$ (weak coupling) or with $V_{2}$ (strong coupling). In other words, both "weak" and "strong" vortices would be created if certain minimum power is injected in the kagome cell. The borderline between the yellowish (light gray) and white regions in Fig. 7 represents the power threshold necessary for generation of a stable "strong" vortex. In general, 


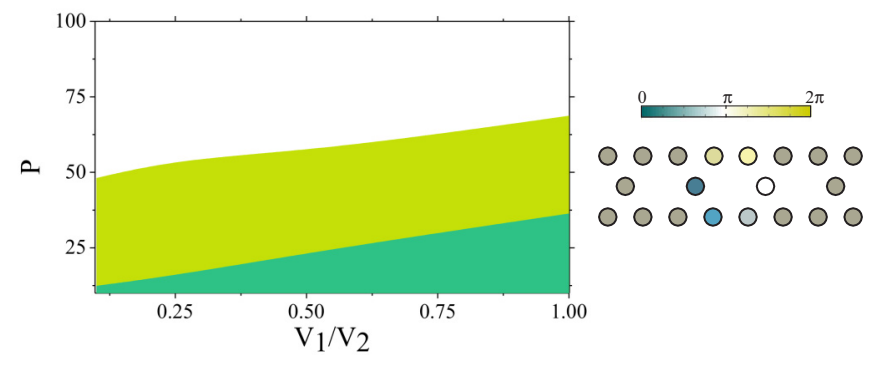

FIG. 7. (Color online) Stability diagram ( $P$ vs $\left.V_{1} / V_{2}\right)$ of vortex ring solutions with $S=1$ bonded between sites with $V_{2}$ ("strong" variant), which are found to exist in the kagome strip. The yellowish (light gray) area represents the power region that corresponds to unstable vortex modes, while the white one depicts the parameter space of stable propagation. The greenish (dark gray) colored area illustrates the region where no vortex modes exist. Phase profile of certain vortex mode $(\beta=-9.91, P=65.6, S=1)$ is shown on the right panel and corresponds to the case of the uniform kagome strip.

the power threshold necessary for the creation of "weak" vortices is lower compared to their "strong counterparts."

In kagome strips, the localized ring-shaped vortex patterns, which in the binary case $V_{1} / V_{2}<1$ can be realized in "weak" and "strong" variants, are stable for a certain range of parameters if they carry angular momentum $S=1$ and 5 (white area in Fig. 7, "strong" variant). They are characterized by the finite existence threshold and oscillatory instability for small powers. In other words, regions of stable propagation correspond to high-power solutions, which is in accordance with direct simulations. Furthermore, the stability region is wider for smaller $V_{1} / V_{2}$. With the increase of $V_{1}$, the power necessary for light localization increases, implying that additional periodicity lowers the excitation threshold for vortices. In the case of kagome ladders, vortex solutions occur for odd topological charges $(S=1,3)$. Unlike kagome strip vortex solutions, these structures are found to be unstable in the whole corresponding existence region.

Particular behavior of phase and amplitude of dynamically stable ring-vortex structures arises from circular flow of energy around a pivot point [41-46]. This property is closely connected with the ability of such structures to carry unchanged the orbital angular momentum and energy to long distances, which can be interesting for technological applications as spatial-division multiplexing (communication lines) and directional transfer of huge energy through the system (high-power lasers). Kagome strips, as settings which support stable vortex propagation in the presence of nonlinearity, can open interesting ways to manipulate light propagation.

Although not specifically emphasized, the saturation tendency of the participation ratio given by Eq. (5) for increasing $z$ is additionally used through the whole paper in order to check the stability of all localized modes' types. Calculations imply that in the case of dynamically stable ring-type solutions, this parameter almost perfectly saturates to a finite value (6 in the case of kagome strip and 4 in the case of ladder configuration).

Finally, we studied the possibility to create moving localized modes in kagome strips and ladders with defocusing nonlinearity. Mathematically, this is done by taking a given stationary solution as the initial condition and multiplying it (a)

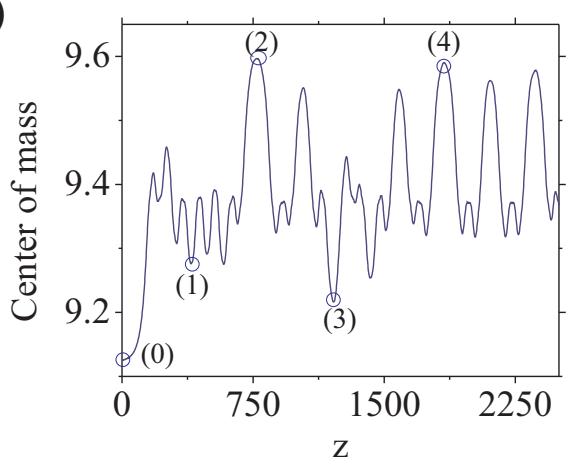

(b)

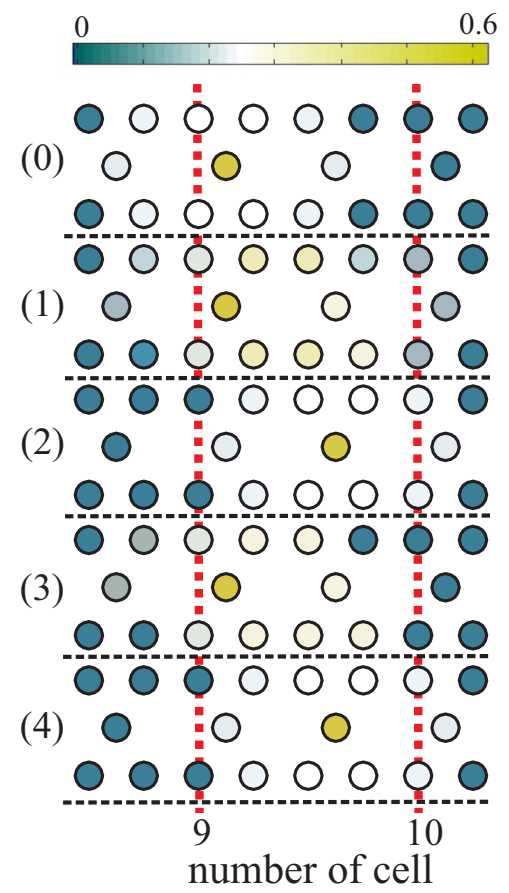

FIG. 8. (Color online) Mobility of the hg mode in the uniform kagome strip configuration. (a) Horizontal center-of-mass evolution of an unstable low-power hg solution $(P=1.02)$ in the presence of a phase gradient $\alpha=0.009$, and (b) intensity profiles taken at different positions in the $z$ direction during the modes' propagation. The corresponding positions are marked in (a) with numbers in brackets.

with a phase gradient factor $\exp \left(-i \alpha p^{\prime}\right)$, where $\alpha$ denotes the strength of the transverse kick and $p^{\prime}$ numbers the unit cells of the uniform chain (with notation of the binary chain in Fig. $1, p^{\prime}=2 p-1$ or $2 p$, respectively). Imposing initial phase gradients in other ways (e.g., allowing phase variations also within unit cells) are found to give qualitatively similar results. Moving modes along a "1D ribbon" direction have been observed in the 2D kagome lattices [27]. These moving modes' shape alternates between "staggered ring" and "hourglass" shape via "intermediate" solution [cf. Figs. 4(a), 4(c), and 4(e) in [27]]. Following this approach, we search for moving modes in the area of stability exchange of the staggered rings and hourglass solutions for a uniform strip, where the two-peak mode was born as a stable solution when the staggered ring branch destabilizes. From the existence curves of the respective modes [Fig. 5(a)] it is possible to notice 
that this area corresponds to a narrow region in the semi-infinite gap near the flat band. In this region hourglass, two-peak, and staggered ring solutions carry approximately the same power for the fixed propagation constant $\beta$. Considering the moving modes, the results showed that, when applying phase kicks to the stationary solutions, the only possibility here is creation of breathing complexes whose central part (center of mass as defined in [27]) oscillatory changes position. In other words, some kind of mobility along the chain consisting in motion of the center of mass between two neighboring ribbon cells: The initial cell and the one next to it [see Figs. 8(a) and 8(b)] was observed. For larger phase kicks, this is more pronounced, e.g., with phase gradient $\alpha=0.8$, a ring mode with $P=1.1$ could be moved one unit cell before getting trapped, but radiating more than in the case with small kick. In general, the mobility in the kagome ribbon is considerably worse than in the 2D case. So, it seems that the dimensional reduction, which in some sense decreases the number of the degrees of freedom in the kagome system, prevents free motion of the localized modes across the ribbons. Figuratively speaking, the decoupling of one ribbon from the rest of the 2D kagome lattice prevents mobility of localized modes across the isolated ribbon. It can be related to the impossibility of radiating (exchanging) energy with the rest of the lattice. The energy transport in the transverse direction with respect to the isolated ribbon is stopped.

For the binary cases, the motion along the chain will be additionally hindered by the fact that neighboring ring modes are alternately strongly and weakly coupled. Since these have quite different properties, no simple path to translate between them exists.

In conclusion, stable propagating nonlinear modes of different power in the semi-infinite gap can be exited by proper arrangement of the lattice parameters, input light beam shape and its strength. Dynamically stable modes with small power possess the staggered ring (strong variant) or hourglasslike shapes and can be observed in both types of the kagome ribbons. For high power, dynamically stable modes of the vortex type can be created in the kagome strips. Unstaggered ring structures (both "weak" and "strong" variants) are also found to be stable in a wide parameter range of the semi-infinite gap. Only the staggered rings formed between the sites with higher coupling constants in binary ribbons bifurcate from the flat band without threshold.

\section{B. Solutions in minigap}

As already stated, binary lattices are characterized by additional small gaps within the band structure (see Fig. 2). Therefore, our analysis includes investigation of nonlinear localized modes that may occur in minigaps, focusing on the first minigap since it is one of the widest gaps and offers the possibility of generating localized solutions that bifurcate from the flat band, if the system exhibits the focusing type of nonlinearity.

The numerical calculations show that the type and properties of the minigap modes depend on the character of nonlinearity. In the focusing nonlinearity case, the branch of staggered ring modes extends from the flat band to the first minigap area. Such rings are shown to be stable in almost the whole region of minigap, which becomes wider for the smaller ratio $V_{1} / V_{2}$. The corresponding existence curves fill the whole region of the minigap, e.g., for $V_{1} / V_{2}=0.25, \beta \in$ $(-2,-1.25)$ (strip) and $\beta \in(-2,-0.5)$ (ladder); $V_{1} / V_{2}=0.5$, $\beta \in(-2,-1.5)$ (strip) and $\beta \in(-2,-1)$ (ladder); $V_{1} / V_{2}=$ $0.75, \beta \in(-2,-1.75)$ (strip) and $\beta \in(-2,-1.5)$ (ladder), as can be seen from Fig. 3 .

In the presence of defocusing nonlinearity, solitons bifurcate from linear modes of the second band. The main characteristic of solutions that bifurcate from this band is their tendency to trap within sites coupled by the smaller coupling coefficient, i.e., $V_{1}$. Part of these modes correspond to the weak staggered rings, which are shown to be unstable in the whole region of minigap.

However, in both kagome configurations, we found several types of exotic localized solutions that preserve their shape (a)

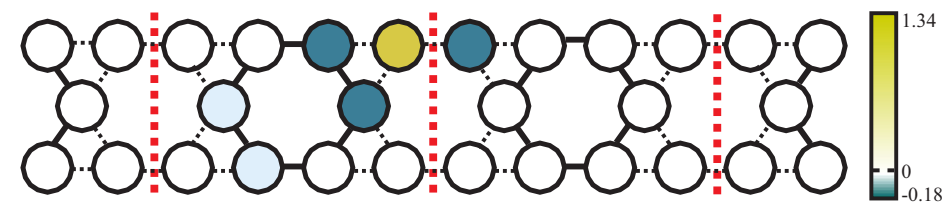

(b)

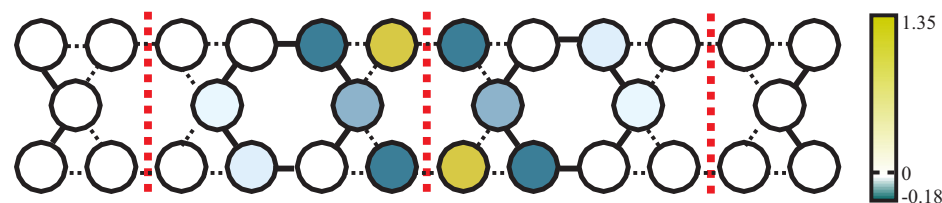

(c)

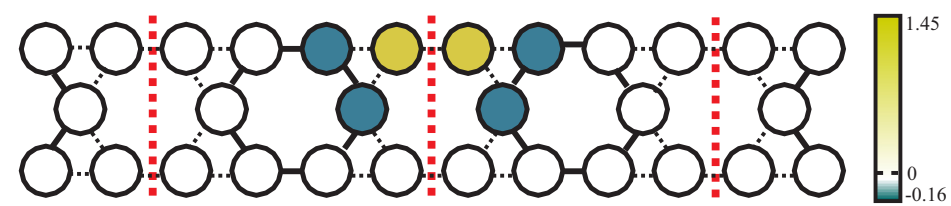

(d)

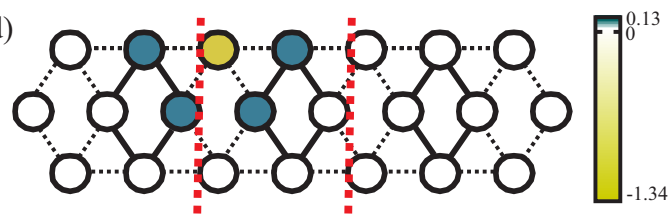

(e)

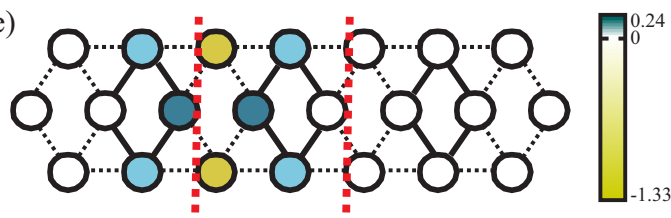

(f)

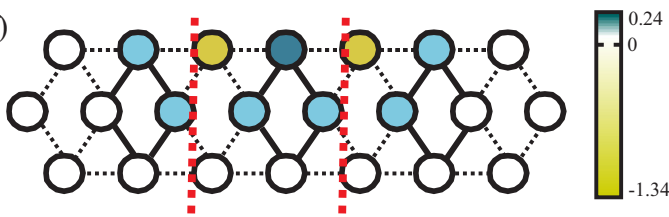

FIG. 9. (Color online) Amplitude profiles of different types of minigap solutions in the binary kagome strip (a)-(c) and binary kagome ladder (d)-(f) ribbon. Red (thick) dashed lines represent boundaries of primitive cells of binary systems. Black solid and dashed (thin) lines stand for different strengths of coupling $V_{2}$ and $V_{1}$, respectively. In all cases, parameters in simulations are set to $V_{1}=0.25$ and $\beta=-1.9$. 
during the propagation for the lattices with strong binarism $V_{1} / V_{2}=0.25$. The maximum of energy of these modes is again concentrated on sites coupled by $V_{1}$. Examples of profiles of different types of such solutions are depicted in Fig. 9. Their robustness during the propagation is due to the width of the minigap which provides less influence of neighboring bands, unlike the case originating in ribbons with $V_{1}=0.5,0.75$. Another feature of minigap solitons is a tendency of their field energy to always localize on the outer site of the ribbon. In both systems, solitons carrying less power are unstable and tend to delocalize.

\section{CONCLUSION}

To conclude, we have theoretically analyzed existence, stability, and dynamical properties of various localized modes in nonlinear binary kagome ribbons. We showed that the first band always stays flat, despite the presence of additional periodicity involved by "binarism." Three types of localized ring solutions are found in the semi-infinite gap of ribbons possessing defocusing nonlinearity: unstaggered ring solitons, staggered ring solitons, and ring vortices. All of them can be localized on the part of the basic binary kagome cell formed of sites coupled stronger between themselves or weaker than with the rest of the sites in the cell. Therefore, we considered "strong" and "weak" variants of nonlinear localized ring modes. The obtained results confirmed the existence of stable unstaggered ring modes in the semi-infinite gap. This is the case for both "strong" and "weak" unstaggered rings and we showed that the binarism increases their stability regime. On the other hand, staggered ring solutions (strong and weak) have proven to be unstable except in a narrow region of the semi-infinite gap near the flat band. At the boundary of this area, the branch of the hourglass localized solution becomes stable. This is recognized as the stability exchange between these two localized solutions. The hourglasslike modes (linear combination of two staggered rings) manifest stable propagation through both considered kagome systems. The main finding is that binarism supports the creation of dynamically stable ring and hourglass modes. Moreover, increasing the "binarism" by lowering the ratio $V_{1} / V_{2}$ favors the robustness of the localized modes. Besides mentioned ring modes, in the semi-infinite gap we found vortex ring solutions with $S \neq 0, M / 2$. In addition, the freely moving localized modes across the kagome ribbons have not been found for any ratio $V_{1} / V_{2}$.

Particularity of the binary kagome ribbons is the opening of the small gaps in the spectrum. Their width increases with lowering the ratio $V_{1} / V_{2}$. We have shown that the nonlinear light localization is possible in these minigaps, too. In the presence of defocusing nonlinearity, the minigap modes are trapped in regions of kagome ribbon cells characterized by smaller coupling parameter $V_{1}$. Their maxima of envelopes are always located on the outer sides of the ribbon, which is not the case for solutions from the semi-infinite gap. The difference in kagome-type geometry influences only the difference in amplitude profiles of certain modes (i.e., number of the solution's peaks), and the stability of vortex solutions. Otherwise, their properties are in general similar. In the kagome ribbon with focusing nonlinearity, only the staggered ring type of minigap solutions can be dynamically stable. Thus, an important conclusion is that binary modulation of the kagome ribbons allows for existence of stable, compact nonlinear modes bifurcating from the linear flat band also for focusing nonlinearity.

Finally, we conclude that control of the light propagation in the kagomelike ribbons is possible by properly arranging the lattice parameters, input light beam shape and strength, which are controllable parameters in experiment. Our findings can be used as a preparatory step for theoretical modelling of new kagomelike structures with particular mode dynamics and experimental investigation on the possibility of realizing and controlling their properties practically at will.

\section{ACKNOWLEDGMENTS}

The authors acknowledge support from the SwedenChilean-Serbian trilateral project "Control of light and matter waves propagation and localization in photonic lattices" from the Swedish Research Council within the Swedish Research Links programme, Grant No. 348-2013-6752, and the Ministry of Education, Science and Technological Development of Republic of Serbia (Project No. III45010). This work was supported in part by Fondecyt Grant No. 1151444, Programa ICM RC-130001, Programa de Financiamiento Basal FB0824.

\section{APPENDIX}

The coupling matrix from Eq. (1) can be represented in the following form:

$$
\tilde{V}=\left(\begin{array}{cccccc}
M_{D} & M_{S}^{T} & 0 & \ldots & 0 & M_{S} \\
M_{S} & M_{D} & M_{S}^{T} & \ldots & 0 & 0 \\
0 & M_{S} & M_{D} & \ldots & 0 & 0 \\
& \vdots & \ddots & & \vdots & \\
0 & 0 & \ldots & M_{S} & M_{D} & M_{S}^{T} \\
M_{S}^{T} & 0 & \ldots & 0 & M_{S} & M_{D}
\end{array}\right),
$$

where in the case of the kagome strip ribbon, the corresponding blocks can be written as

$$
M_{D}=\left(\begin{array}{cccccccccc}
0 & V_{1} & 0 & 0 & V_{1} & 0 & 0 & 0 & 0 & 0 \\
V_{1} & 0 & V_{2} & 0 & V_{2} & 0 & 0 & 0 & 0 & 0 \\
0 & V_{2} & 0 & V_{1} & 0 & V_{2} & 0 & 0 & 0 & 0 \\
0 & 0 & V_{1} & 0 & 0 & V_{1} & 0 & 0 & 0 & 0 \\
V_{1} & V_{2} & 0 & 0 & 0 & 0 & V_{1} & V_{2} & 0 & 0 \\
0 & 0 & V_{2} & V_{1} & 0 & 0 & 0 & 0 & V_{2} & V_{1} \\
0 & 0 & 0 & 0 & V_{1} & 0 & 0 & V_{1} & 0 & 0 \\
0 & 0 & 0 & 0 & V_{2} & 0 & V_{1} & 0 & V_{2} & 0 \\
0 & 0 & 0 & 0 & 0 & V_{2} & 0 & V_{2} & 0 & V_{1} \\
0 & 0 & 0 & 0 & 0 & V_{1} & 0 & 0 & V_{1} & 0
\end{array}\right),
$$

and $M_{S}$ is a sparse $10 \times 10$ matrix, with nonzero elements $M_{S}(1,4)=M_{S}(7,10)=V_{1}$.

Similarly, the nearest-neighbor coupling in the ladder configuration can be represented via a matrix with the following 
block matrices:

$$
M_{D}=\left(\begin{array}{cccccc}
0 & V_{1} & V_{1} & 0 & 0 & 0 \\
V_{1} & 0 & V_{2} & V_{2} & 0 & 0 \\
V_{1} & V_{2} & 0 & 0 & V_{1} & V_{2} \\
0 & V_{2} & 0 & 0 & 0 & V_{2} \\
0 & 0 & V_{1} & 0 & 0 & V_{1} \\
0 & 0 & V_{2} & V_{2} & V_{1} & 0
\end{array}\right),
$$

and $M_{S}$ is now a sparse $6 \times 6$ matrix, with nonzero elements $M_{S}(1,2)=M_{S}(1,4)=M_{S}(5,4)=M_{S}(5,6)=V_{1}$.

[1] J. D. Joannopoulos, S. G. Johnson, J. N. Winn, and R. D. Meade, Photonic Crystals: Molding the Flow of Light, 2nd ed. (Princeton University Press, Princeton, 2008).

[2] P. Yeh, A. Yariv, and C. S. Hong, J. Opt. Soc. Am. 67, 423 (1977).

[3] M. Segev, Y. Silberberg, and D. N. Christodoulides, Nature Photon. 7, 197 (2013).

[4] Z. Valy Vardeny, A. Nahata, and A. Agrawal, Nature Photon. 7, 177 (2013).

[5] N. Akhmediev, J. M. Dudley, D. R. Solli, and S. K. Turitsyn, J. Opt. 15, 060201 (2013).

[6] I. V. Barashenkov, L. Baker, and N. V. Alexeeva, Phys. Rev. A 87, 033819 (2013).

[7] N. G. R. Broderick, C. M. de Sterke, and B. J. Eggleton, Phys. Rev. E 52, R5788(R) (1995).

[8] A. Kanshu, C. E. Rüter, D. Kip, V. M. Shandarov, P. P. Beličev, I. Ilić, and M. Stepić, Opt. Lett. 37, 1253 (2012).

[9] T. X. Tran, X. N. Nguyen, and D. C. Duong, J. Opt. Soc. Am. B 31, 1132 (2014).

[10] H. Tasaki, Eur. Phys. J. B 64, 365 (2008).

[11] D. L. Bergman, C. Wu, and L. Balents, Phys. Rev. B 78, 125104 (2008).

[12] L. Zheng, L. Feng, and W. Yong-Shi, Chin. Phys. B 23, 077308 (2014).

[13] J. Vidal, R. Mosseri, and B. Doucot, Phys. Rev. Lett. 81, 5888 (1998).

[14] D. Leykam, S. Flach, O. Bahat-Treidel, and A. S. Desyatnikov, Phys. Rev. B 88, 224203 (2013).

[15] J. D. Bodyfelt, D. Leykam, C. Danieli, X. Yu, and S. Flach, Phys. Rev. Lett. 113, 236403 (2014).

[16] T. Neupert, L. Santos, C. Chamon, and C. Mudry, Phys. Rev. Lett. 106, 236804 (2011).

[17] E. J. Bergholtz and Z. Liu, Int. J. Phys. B 27, 1330017 (2013).

[18] M. C. Rechtsman, J. M. Zeuner, Y. Plotnik, Y. Lumer, D. Podolsky, F. Dreisow, S. Nolte, M. Segev, and A. Szameit, Nature (London) 496, 196 (2013).

[19] M. Hafezi, S. Mittal, J. Fan, A. Migdall, and J. Taylor, Nature Photon. 7, 1001 (2013).

[20] Z. Wang, Y. Chong, J. D. Joannopoulos, and M. Soljacic, Nature (London) 461, 772 (2009).

[21] D. Leykam, O. Bahat-Treidel, and A. S. Desyatnikov, Phys. Rev. A 86, 031805(R) (2012).

[22] R. A. Vicencio, C. Cantillano, L. Morales-Inostroza, B. Real, C. Mejía-Cortés, S. Weimann, A. Szameit, and M. I. Molina, Phys. Rev. Lett. 114, 245503 (2015); D. Guzmán-Silva, C. MejiáCorteś, M. A. Bandres, M. C. Rechtsman, S. Weimann, S. Nolte,
M. Segev, A. Szameit, and R. A. Vicencio, New J. Phys. 16, 063061 (2014).

[23] S. Mukherjee, A. Spracklen, D. Choudhury, N. Goldman, P. Öhberg, E. Andersson, and R. R. Thomson, Phys. Rev. Lett. 114, 245504 (2015).

[24] M. Boguslawski, P. Rose, and C. Denz, Appl. Phys. Lett. 98, 061111 (2011).

[25] R. A. Vicencio and C. Mejía-Cortés, J. Opt. 16, 015706 (2014).

[26] K. J. H. Law, A. Saxena, P. G. Kevrekidis, and A. R. Bishop, Phys. Rev. A 79, 053818 (2009).

[27] R. A. Vicencio and M. Johansson, Phys. Rev. A 87, 061803(R) (2013).

[28] I. Syôzy, Prog. Theor. Phys. 6, 306 (1951).

[29] L. Wang, B. Terhalle, V. A. Guzenko, A. Farhan, M. Hojeij, and Y. Ekinci, Appl. Phys. Lett. 101, 093104 (2012).

[30] J. Ruostekoski, Phys. Rev. Lett. 103, 080406 (2009).

[31] G.-B. Jo, J. Guzman, C. K. Thomas, P. Hosur, A. Vishwanath, and D. M. Stamper-Kurn, Phys. Rev. Lett. 108, 045305 (2012).

[32] Y. Nakata, T. Okada, T. Nakanishi, and M. Kitano, Phys. Rev. B 85, 205128 (2012).

[33] M. I. Molina, Phys. Lett. A 376, 3458 (2012).

[34] S. R. White and R. R. P. Singh, Phys. Rev. Lett. 85, 3330 (2000).

[35] P. Azaria, C. Hooley, P. Lecheminant, C. Lhuillier, and A. M. Tsvelik, Phys. Rev. Lett. 81, 1694 (1998).

[36] Ch. Waldtmann, H. Kreutzmann, U. Schollwöck, K. Maisinger, and H.-U. Everts, Phys. Rev. B 62, 9472 (2000).

[37] K. Davies, K. Miura, N. Sugimoto, and K. Hirao, Opt. Lett. 21, 1729 (1996).

[38] K. Itoh, W. Watanabe, S. Nolte, and C. Schaffer, MRS Bull. 31, 620 (2006).

[39] A. Szameit and S. Nolte, J. Phys. B 43, 163001 (2010).

[40] O. Derzhko, J. Richter, A. Honecker, M. Maksymenko, and R. Moessner, Phys. Rev. B 81, 014421 (2010).

[41] D. E. Pelinovsky, P. G. Kevrekidis, and D. J. Frantzeskakis, Physica D 212, 20 (2005).

[42] D. N. Neshev, T. J. Alexander, E. A. Ostrovskaya, Yu. S. Kivshar, H. Martin, I. Makasyuk, and Z. Chen, Phys. Rev. Lett. 92, 123903 (2004).

[43] J. W. Fleischer, G. Bartal, O. Cohen, O. Manela, M. Segev, J. Hudock, and D. N. Christodoulides, Phys. Rev. Lett. 92, 123904 (2004).

[44] B. Luther-Davies, J. Christou, V. Tikhonenko, and Yu. S. Kivshar, J. Opt. Soc. Am. B 14, 3045 (1997).

[45] S. N. Khonina, N. L. Kazanskiy, and V. A. Soifer, Optical Vortices in a Fiber: Mode Division Multiplexing and Multimode Self-Imaging, Recent Progress in Optical Fiber Research, edited 
by M. Yasin, S. W. Harun, and H. Arof (INTECH, Rijeka, Croatia, 2012).

[46] Lj. Hadžievski, A. Maluckov, A. M. Rubenchik, and S. Turitsyn, Light: Science \& Applications 4, e314 (2015).

[47] F. Lederer, G. I. Stegeman, D. N. Christodoulides, G. Assanto, M. Segev, and Y. Silberberg, Phys. Rep. 463, 1 (2008).
[48] Z. Chen, M. Segev, and D. N. Christodoulides, Rep. Prog. Phys. 75, 086401 (2012).

[49] G. Gligorić, A. Maluckov, Lj. Hadžievski, and B. A. Malomed, Phys. Rev. A 78, 063615 (2008).

[50] W. H. Press, S. A. Teukolsky, W. T. Vetterling, and B. P. Flannery, Numerical Recipes: The Art of Scientific Computing, 3rd ed. (Cambridge University Press, New York, 2007). 